\title{
Gehörgangsatresie wann operieren?
}

Frage: Ich betreue eine dreijährige $\mathrm{Pa}$ tientin mit Gehörgangsatresie. Wann wäre der richtige Zeitpunkt, um sie zu operieren?

Antwort: Bei Patienten mit Gehörgangsatresie (GGA) ist die Innenohrfunktion aufgrund des unterschiedlichen entwicklungsgeschichtlichen Ursprungs meist normal, jedoch entsteht durch den Verschluss des Gehörgangs eine Schallleitungsschwerhörigkeit von bis zu $60 \mathrm{~dB}$. Bei der Beantwortung der oben genannten Frage müssen zwei Gesichtspunkte berücksichtigt werden: die funktionelle und die kosmetische Rehabilitation.

Bei der funktionellen Rehabilitation sind Patienten mit einer einseitigen GGA von Patienten mit einer beidseitigen GGA zu unterscheiden. Während einseitig betroffene Patienten auch unversorgt $\mathrm{zu}$ nächst eine normale Laut- und Sprachentwicklung zeigen, müssen beidseitig betroffene Patienten so früh wie möglich mit Knochenleitungshörsystemen (KLHS) versorgt werden, bis eine definitive (operative) Therapie durchgeführt werden kann. Patienten mit einseitiger GGA und Normalhörigkeit der Gegenseite wurden aufgrund der normalen Sprachentwicklung lange Zeit nicht mit Hörgeräten versorgt. Erst Ende des letzten Jahrhunderts wurde erkannt, dass bei diesen Patienten im unversorgten Zustand doch erhebliche Probleme beim räumlichen Hören oder beim Hören im Störgeräusch auftreten und einseitig schwerhörige Kinder zum Beispiel öfter eine Schulklasse wiederholen müssen [2], so dass auch bei Patienten mit einseitiger GGA schon im ersten Lebensjahr eine Versorgung mit einem KLHS zu empfehlen ist.

Zur definitiven funktionellen Versorgung für Kinder stehen neben implantierbaren KLHS auch aktive Mittelohrhörsysteme (AMHS) zur Verfügung. Für welches System der einzelne Patient geeignet ist oder ob auch eine operative Gehörgangsplastik mit Mittelohrrekonstruktion sinnvoll möglich ist, ist unter anderem von den anatomischen Gegebenheiten abhängig. Mittels eines hochauflösenden Felsenbein-CT kann der Jahrsdoerfer-Score bestimmt werden [3]. Bei Werten unter 4 sollte eine Versorgung mit einem KLHS erfolgen, bei Werten von 4 und mehr kann ab dem zweiten Lebensjahr über die Implantation eines AMHS diskutiert werden. Bei Patienten mit Werten von 7 und mehr kann auch über eine spätere operative Gehörgangsanlage mit Mittelohrrekonstruktion nachgedacht werden.

Die Erfahrung hierbei zeigt allerdings eine hohe Re-Stenosierungsrate, wiederholte operative Eingriffe und die Notwendigkeit, trotz erfolgreicher Operation in mehr als $30 \%$ der Fälle doch weiter ein Hörgerät tragen zu müssen. Die sicherste funktionelle Rehabilitation bei geringem

\section{Haben Sie auch eine fachliche Frage?}

Das "Consilium HNO" ist ein Service des Unternehmens Infectopharm. Als HNO-Arzt haben Sie die Möglichkeit, selbst Fragen zum gesamten Fachgebiet zu stellen. Schreiben Sie dazu an Dr. Kristin Brendel-Walter, Von-Humboldt-Straße 1, 64646 Heppenheim, oder schicken Sie eine E-Mail an consilium@infectopharm.com. Ihre Frage wird anonym an ein Mitglied des Expertenpools weitergeleitet, Sie erhalten eine schriftliche Antwort.

Wenn Sie sehen wollen, was die Kolleginnen und Kollegen so fragen, können Sie im Archiv des "Consilium HNO" unter www.infectopharm.com/consilium stöbern. Außerdem werden redaktionell ausgewählte, allgemein interessante Anfragen zusätzlich in der Zeitschrift „HNO-Nachrichten“ publiziert.
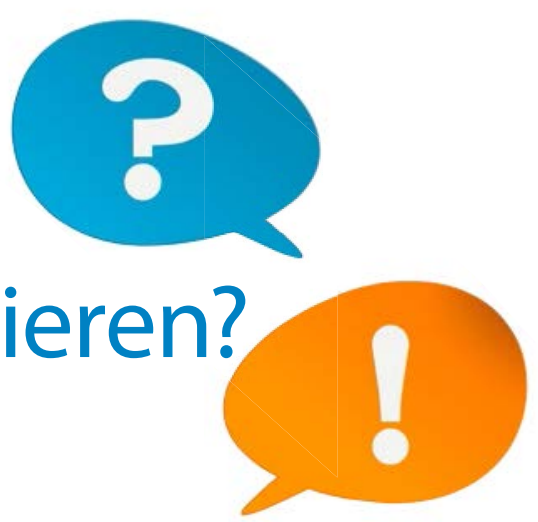

operativem Aufwand ist mit einem KLHS $\mathrm{zu}$ erzielen. Hierbei ist allerdings zu berücksichtigen, dass durch KLHS im Gegensatz zu AMHS beide Seiten stimuliert werden, was in bestimmten Hörsituationen dann oft zu Interferenzen führt, sodass aus funktioneller Sicht eine Rehabilitation mit einem AMHS anzustreben ist, wenn dies anatomisch erfolgsversprechend erscheint $[4,5]$.

Die kosmetische Rehabilitation ist auf unterschiedliche Arten möglich. Neben magnetgetragenen, knochenverankerten sowie Klebe-Epithesen kann eine vollständige chirurgische Rekonstruktion aus autologem, körpereigenem Material (v. a. Rippenknorpel) oder mit Unterstützung von Implantaten aus porösem Polyethylen [6] angestrebt werden. Hier ist eine eingehende Beratung der Patienten und Eltern über die jeweiligen Vor- und Nachteile unter Berücksichtigung des teils erheblichen operativen Aufwands, wiederholter Operationen, möglicher Hebedefekte und die Erwartungshaltung aller Beteiligten zu besprechen.

Im vorliegenden Fall kann somit bei Auftreten von funktionellen Defiziten in den regelmäßigen Verlaufskontrollen bei einseitiger GGA in Abhängigkeit von den anatomischen Voraussetzungen eine funktionelle Rehabilitation mit einem aktiven Mittelohrhörsystem oder einem Knochenleitungshörsystem angeraten werden. Bei gleichzeitig vorliegender Mikrotie oder Dysplasie der Ohrmuschel kann eine einzeitige ästhetische Rehabilitation mit einer epithetischen Versorgung oder einer chirurgischen Ohrmuschelrekonstruktion mit Implantaten aus Polyethylen oder Rippenknorpel erfolgen.

\section{Literatur beim Verfasser}

\section{Dr. med. Stefan Volkenstein}

St. Elisabeth-Hospital

Universitätsklinik für Hals-,Nasen- und Ohrenheilkunde, Kopf- und Hals-Chirurgie Bleichstr. 15, 44787 Bochum 